\title{
Selective nucleation and controlled growth: quantum dots on metal, insulator and semiconductor surfaces
}

\author{
By J. A. Venables $S^{1,4}$, P. A. BennetT 1 , H. BRune 2 , \\ J. DRUCKER ${ }^{1}$ AND J. H. HARDING ${ }^{3}$ \\ ${ }^{1}$ Department of Physics and Astronomy, Arizona State University, \\ PO Box 871504, Tempe, AZ 85287-1504, USA (john.venables@asu.edu) \\ ${ }^{2}$ Institut de Physique des Nanostructures, Faculté des Sciences de Base, \\ Ecole Polytechnique Fédérale de Lausanne, \\ 1015 Lausanne-Ecublens, Switzerland \\ ${ }^{3}$ Department of Physics and Astronomy, University College London, \\ Gower Street, London WC1E 6BT, UK \\ ${ }^{4}$ School of Chemistry, Physics and Environmental Science, \\ Chichester Building, University of Sussex, Brighton BN1 9QJ, UK
}

\section{Published online 18 December 2002}

Nucleation and growth models are well developed for nucleation on homogeneous substrates, and they can typically be described in terms of three energy parameters. Nucleation on substrates containing point-defect traps has been investigated, at the cost of introducing more energy parameters. This paper outlines the quantitative description of such growth models, using rate and rate-diffusion equations, in terms of energies for individual surface processes, with examples taken from metalmetal, metal-insulator and semiconductor growth. The challenge to modelling is to describe the large range of length and time-scales in thin-film fabrication and degradation, without relying on too many (unknown) material parameters, which often occur in combination. Separating them into elementary processes often proves to be a challenge. One typically requires selective nucleation using patterned substrates, in combination with controlled, self-organized, growth for reliable nanotechnology. Reconstructed semiconductor surfaces offer both a further challenge to modelling and an opportunity for future technology; these paradoxes are discussed briefly.

Keywords: nucleation and growth; rate equations; rate-diffusion equations; metal growth; patterned substrates; semiconductor growth

\section{Introduction}

Nucleation and growth on surfaces has been studied intensively over the last 30 years. In the specific case of deposition from the vapour, it is well known that individual atomic events can strongly influence and even dominate the final micro- or nanostructure of epitaxial thin films (Venables 1994, 2000). Scanning-tunnelling-microscopy (STM) (Brune 1998; Bennett \& von Känel 1999) and field-ion-microscopy (FIM)

One contribution of 13 to a Discussion Meeting 'Quantum dots: science on the smallest scale?'. 
(Ehrlich 1991, 1994; Kellogg 1994; Tsong 1990) experiments are able to follow such individual events. Such experiments, which exploit the surface sensitivity and ultrahigh resolution of the STM and FIM, have mostly been carried out on clean, lowindex metal surfaces at low temperatures, and they have emphasized the extreme kinetic limit, where the main process is surface diffusion by individual adatoms and pairs of adatoms are already stable clusters. Analysis of such experiments has led to accurate values of the adatom diffusion coefficient $D_{1}$ and diffusion energy $\left(E_{\mathrm{d}}\right.$ and $E_{\mathrm{m}}$ are used in the literature), which have been tabulated in several cases in the above references.

On the other hand, experiments at higher temperature have required more parameters for their interpretation, but the order of events depends on the system studied. For metals deposited onto insulators such as alkali halides, the adatom adsorption energy $E_{\mathrm{a}}$ is typically the first additional parameter needed, as the metal adatom is rather weakly bonded to the substrate. In this case re-evaporation can take place at moderate temperatures, and the mean free path for adatoms is determined by $\left(E_{\mathrm{a}}-E_{\mathrm{d}}\right)$. For other systems, such as metal or semiconductor growth, $\left(E_{\mathrm{a}}-E_{\mathrm{d}}\right)$ is relatively high, so that the first process to intervene may be the break-up of small clusters, which can be parametrized by a lateral binding energy $E_{\mathrm{b}}$ between adatoms. Experiments on all these systems have been carried out by the full range of surface analytical and microscopical techniques, including, where practical, in situ observations of the nucleation and growth dynamics at the growth temperature. However, many growth methods, for example the widely used chemical vapour deposition (CVD), are much too complex for this approach to work; we have to rely almost entirely on ex situ observations, taken after growth at elevated temperature and subsequent cooling, typically to room temperature.

There are three main types of model that are used to connect these experiments to atomic-level parameters. The first, rate equations (REs) and rate-diffusion equations, has a long history in chemical kinetics; this largely deterministic approach is emphasized here. The second, kinetic Monte Carlo (KMC) simulation, incorporates statistical fluctuations, and is widely used. The advantage of direct simulation is that all processes thought to be important can be included, but the corresponding disadvantages are that the rates of all such processes must be explicitly included, and the computational effort scales with the rate constant of the fastest process, typically adatom diffusion. The problem is even more acute for molecular dynamics (MD) simulations, where individual atomic motions are followed in real time. The level set (LS) method (Petersen et al. 2001; Ratsch et al. 2002) has been developed more recently. In particular, the LS method combines an atomic-level description in the direction perpendicular to the substrate, with a deterministic continuum description in the substrate plane; it is therefore highly appropriate for investigating layer growth.

Any of these methods can be used to determine atomic-level parameters, or a combination of parameters, which are needed to describe particular experimental results. Comparison of such parameters with $a b$ initio quantum theory is then a reasonable goal, which has been successfully achieved in a few cases. Once this circle has been closed we can be satisfied and move onto other problems. However, it remains a major challenge to do this in general. Crystal growth on substrates is an archetypal problem for multi-scale modelling, involving a large range of length- and time-scales. An individual method can only examine a very restricted subset of all such scales. In 


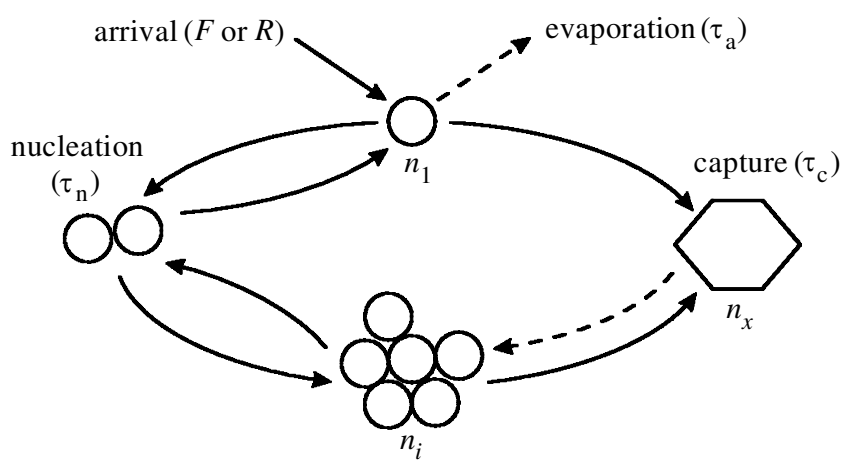

Figure 1. Schematic of the interaction between nucleation and growth stages. The adatom density $n_{1}$ determines the critical cluster density $n_{i}$; however, $n_{1}$ is itself determined by the arrival flux or rate $(F$ or $R$ ) in conjunction with the various loss processes, which have associated characteristic times $\left(\tau_{\mathrm{a}}, \tau_{\mathrm{n}}\right.$ and $\left.\tau_{\mathrm{c}}\right)$ as described in the text. (After Venables $(1987,2000)$.)

particular, the growth of quantum dots (QDs) on (reconstructed, compound) semiconductor surfaces involves a whole series of reactions and potentially rate-limiting steps, which has presented a serious challenge to thorough analysis. We return to this topic in $\S 5$. But first, in $\S 2$, the progress that has been made in describing nucleation and growth using REs is described. Then, in $\S 3$, nucleation on point-defect sites is outlined; such defects can form the basis of a nanofabrication technique involving self-assembly. Section 4 deals with rate-diffusion equations in one and two dimensions, illustrated with recent experimental examples.

\section{Rate equations and algebraic solutions}

Rate equations are a natural starting point in the modelling of atomic, molecular and electronic processes. In the case of the processes discussed in $\S 1$ for cluster growth on surfaces, adatoms or admolecules are typically the mobile species, and we need to write down the REs for these species and for their incorporation into clusters of all sizes and configurations. Needless to say, key simplifications are required to make progress.

A useful, but rather drastic, approximation is to divide the infinite set of REs into three sharp categories. The first category is the single adatom areal density, for which the RE can be written as

$$
\frac{\mathrm{d} n_{1}}{\mathrm{~d} t}=F(1-Z)-\frac{n_{1}}{\tau}, \quad \tau^{-1}=\tau_{\mathrm{a}}^{-1}+\tau_{\mathrm{n}}^{-1}+\tau_{\mathrm{c}}^{-1}+\cdots .
$$

Here $F$ is the deposition flux (or equivalently the rate $R$ ) and $Z$ is the coverage of the substrate by stable clusters. The composite term $n_{1} / \tau$ represents all the loss terms, adsorption, nucleation, capture by stable clusters and maybe others (hence the dots), which add like resistances in parallel. In the second category, we consider the statistical mechanics of small clusters of size $j$, via the Walton relation (see Venables $1987,1994,2000)$. We can then show that the corresponding rate equations for $n_{j}$ are all effectively zero, and that $n_{j}$ is proportional to $n_{1}^{j}$; these equations represent subcritical clusters, $2 \leqslant j \leqslant i$, where $i$ is the critical cluster size. If $i=1$, there are no such clusters or equations, at high supersaturation. Finally, all larger clusters $(j>i)$ 
are deemed to be stable, and are grouped together as a density $n_{x}$ in one further equation

$$
\frac{\mathrm{d} n_{x}}{\mathrm{~d} t}=U_{i}-U_{\mathrm{c}},
$$

where the nucleation rate $U_{i}=\sigma_{i} D_{1} n_{1} n_{i}$, where $\sigma_{i}$ is the relevant capture number, discussed further in $\S 4$. The coalescence rate, $U_{\mathrm{c}}$, has been expressed as $U_{\mathrm{c}}=2 n_{x} \mathrm{~d} Z / \mathrm{d} t$, although other forms become relevant at high coverage (Brune et al. 1999). The competitive nature of the processes discussed above is illustrated schematically in figure 1 (Venables 1987).

The above simplifications mean that we have reduced our formulation to two coupled, nonlinear, ordinary differential equations (ODEs) for $n_{1}$ and $n_{x}$. We now have two tasks. Our first task is to input a sensible set of processes. Capture by stable clusters is important in limiting nucleation, giving rise to the term $\tau_{\mathrm{c}}^{-1}$ in equation (2.1); we can write this as $\tau_{c}^{-1}=\sigma_{x} D_{1} n_{x}$. It is clear from this expression, and the schematic of it in figure 1, how the nonlinearities arise; $n_{x}$ depends on the $(j+1)$ th power of $n_{1}$ via equation $(2.2)$, but $n_{x}$ is also involved in limiting $n_{1}$ in equation (2.1). Depending on the processes that dominate, different regimes are expected, and if further processes are included (cluster mobility or mobilityinduced coalescence for example), they may lead to different power laws and different temperature dependencies. These points have long been appreciated in the literature (e.g. Venables 1973), and later examples may also be found via references quoted here.

The second task is to adopt a particular method of solution. We can simply integrate the differential equations, casting them in matrix form if appropriate; clearly we are not limited to two equations as above, but using more risks becoming unduly dependent on unknown parameters, which may or may not be included in a consistent fashion. These ODEs are stiff, and instabilities can easily result from using time or coverage steps that are too large. Some examples of direct integration are given in $\oint 4$.

A second method of solution is to make a steady-state approximation, in which all net rates of change are zero. For example, after an initial transient time, which is shorter than any of the individual times making up the overall $\tau$ in equation (2.1), $\mathrm{d} n_{1} / \mathrm{d} t$ is effectively zero. Similarly, if we limit our attention to the maximum in $n_{x}, \mathrm{~d} n_{x} / \mathrm{d} t$ is also zero. Thus, in this case, the ODEs reduce to nonlinear coupled algebraic equations, for which we can obtain explicit or iterative solutions.

These algebraic solutions allow one to extract all the relevant material parameter dependencies within any one condensation regime, and they are not limited to particular values of the critical nucleus size, $i$, as has been assumed by particular authors on particular occasions. The value of $i$ is that value of an assumed $j$ which yields the minimum nucleation rate or density. During direct integration, this $i$-value can vary, thus including phenomena such as Ostwald ripening during deposition or annealing. In an algebraic solution, it is the value that produces the minimum density $n_{x}$ and so corresponds to the effective value for the deposition as a whole under the given conditions. The important point is that the critical nucleus size is an output of the calculation that has characteristic energies as input, not the other way around.

Modern computational packages can make these points conceptually clear. As illustrated in figure 2, MATLAB ${ }^{\mathrm{TM}} 5.3$ (Student edition) has been used to model the rela- 

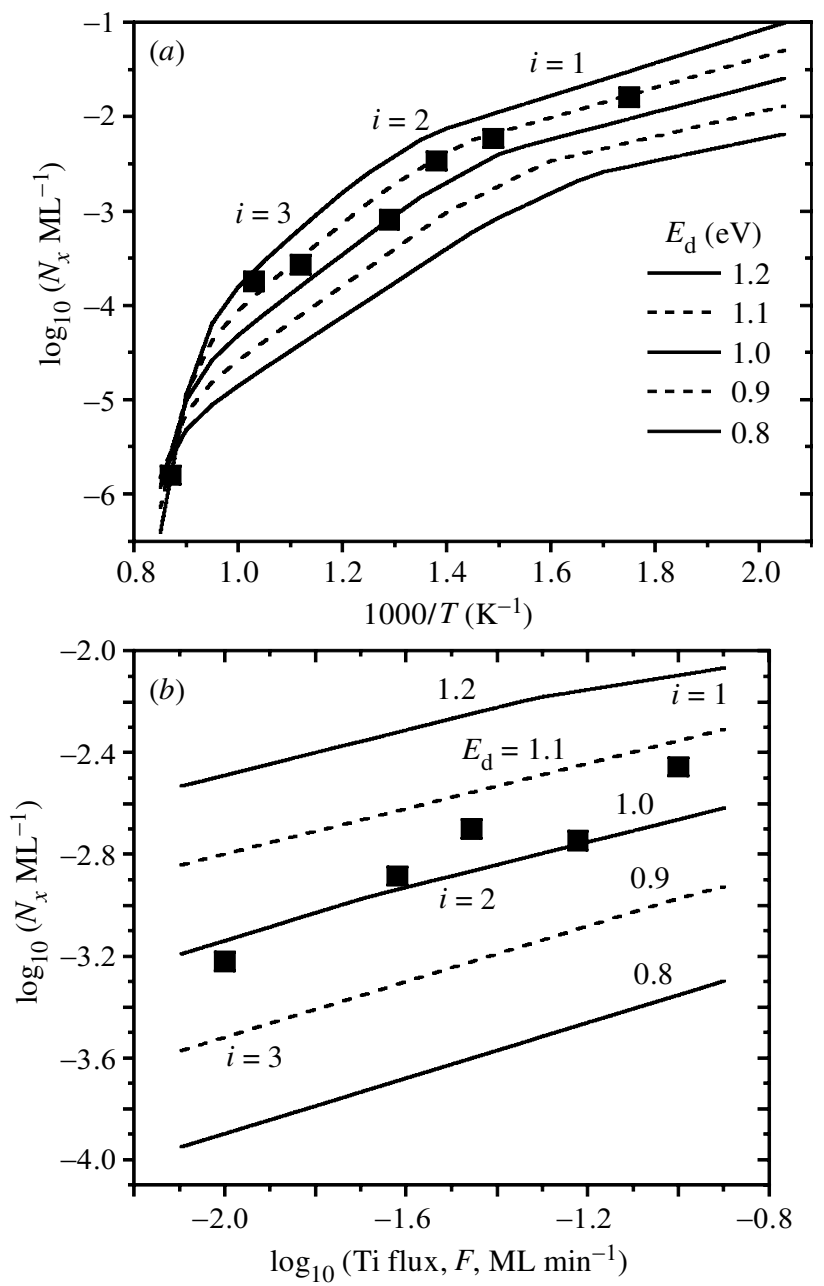

Figure 2. Algebraic solution to REs for $E_{\mathrm{a}}=2.55, E_{\mathrm{b}}=0.7 \mathrm{eV}$ and $E_{\mathrm{d}}=0.8-1.2 \mathrm{eV}$. (a) Temperature dependence for $F=0.06 \mathrm{ML} \mathrm{min}^{-1}$; $(b)$ flux dependence for $T=450{ }^{\circ} \mathrm{C}$. Pair-binding model for $E_{j}$ up to $j=3$, but with $E_{3}=2.2 E_{\mathrm{b}}$, rather than $2 E_{\mathrm{b}}$, which allows a range for $i=2$ that would otherwise be absent (this range is longer the higher $E_{3}$ is above $2 E_{\mathrm{b}}$ ). Extension to higher $i$-values might require higher values of $E_{\mathrm{a}}$ for comparable agreement at high-temperature in (a). Experimental nucleation densities for $\mathrm{Ti} / \mathrm{Si}(001)$ are taken from McDaniels et al. (2001) and show agreement with $E_{\mathrm{b}}=1.1 \pm 0.1 \mathrm{eV}$. See text for discussion.

tively complex surface reaction of $\mathrm{Ti}$ deposited onto $\mathrm{Si}(001)$ during the initial stages of titanium silicide $\left(\mathrm{TiSi}_{2}\right)$ formation. This system has been investigated experimentally by several techniques, and Arrhenius $\left(T^{-1}\right)$ and power-law $F$-dependencies are those expected for simple nucleation and growth models (McDaniels et al. 2001), with a small value of $i$ in the range $2-6$. The algebraic solution is formulated as a matrix in $\left[T^{-1}, j\right]$, which is simultaneously addressed, to arrive at an iterative solution dependent on the three energies $E_{\mathrm{d}}, E_{\mathrm{b}}$ (which is built up into $E_{j}$ via pair-bond arguments) and $E_{\mathrm{a}}$ (which intervenes only at the highest $T$-values). In this case, we can obtain a perfect fit to a relatively small dataset, with the energy values given 
(a)

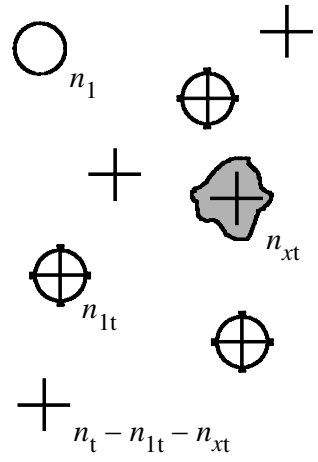

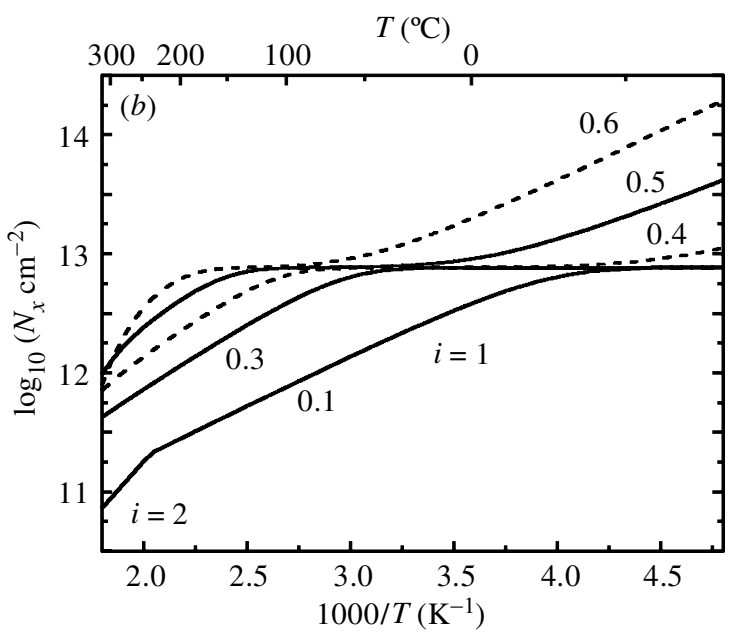

Figure 3. (a) Model for nucleation at attractive random point defects (density $n_{\mathrm{t}}$ ), which can be occupied by adatoms (density $n_{1 \mathrm{t}}$ ), clusters (density $n_{x \mathrm{t}}$ ) or can be empty. (b) Algebraic solution to rate equations for trapping energy $E_{\mathrm{t}}=0.5 \mathrm{eV}, E_{\mathrm{a}}=1.16 \mathrm{eV}, E_{\mathrm{b}}=1.04 \mathrm{eV}$ and $E_{\mathrm{d}}=0.1-0.6 \mathrm{eV}$. Originally used for experiments on $\mathrm{Fe} / \mathrm{CaF}_{2}(111)$ (Heim et al. 1996) and recalculated for Venables (2000). See text for discussion.

in the figure, and the whole exercise takes less than a minute on a $300 \mathrm{MHz}$ laptop computer. Algebraic solutions are very quick, faster than integrating ODEs, and much faster than KMC or LS methods. Thus, they have an educative value, and can provide an overall summary of the behaviour, provided that one accepts the conditions that lead to the formulation in the first place.

Does this mean that we believe the fine details of this model, as applied to the complex, reactive system $\mathrm{Ti}+2 \mathrm{Si} \rightarrow \mathrm{TiSi}_{2}$ ? No, we do not: what figure 2 shows is that the RE pair-binding formulation is sufficiently flexible to provide an adequate three-parameter fit to a limited dataset and that, in the situations illustrated, the supply of $\mathrm{Si}$ is not rate limiting. It is thought that Ti may diffuse as subsurface interstitials, creating mobile $\mathrm{Si}$ ad-dimers and immobile dimer vacancies. The late transition metals ( $\mathrm{Co}, \mathrm{Ni}, \mathrm{Pd}$, etc.) dissolve into the bulk rather than re-evaporate (McDaniels et al. 2001), so $E_{\text {a }}$ may not represent re-evaporation in this case. So the energy parameters, obtained by comparison of the model with experiment, are often lumped, and may be open to interpretation. Moreover, one needs several sets of independent experimental data to test the model in detail, and these may not be easy to obtain. These points apply with even greater force when we consider extending the models beyond three energy parameters.

\section{Extensions to defect nucleation}

In many cases, particularly of metals deposited onto insulators, it has long been known that surface point defects and line defects such as surface steps act as preferred nucleation sites (see Venables 1994, 2000 for references). More recently, this aspect has become of interest for nanofabrication, since well-defined and positioned traps are possible routes to the self-assembly of nanostructures. Only point-defect traps, which necessarily introduce two extra parameters, a trapping energy, $E_{\mathrm{t}}$, and a trap 

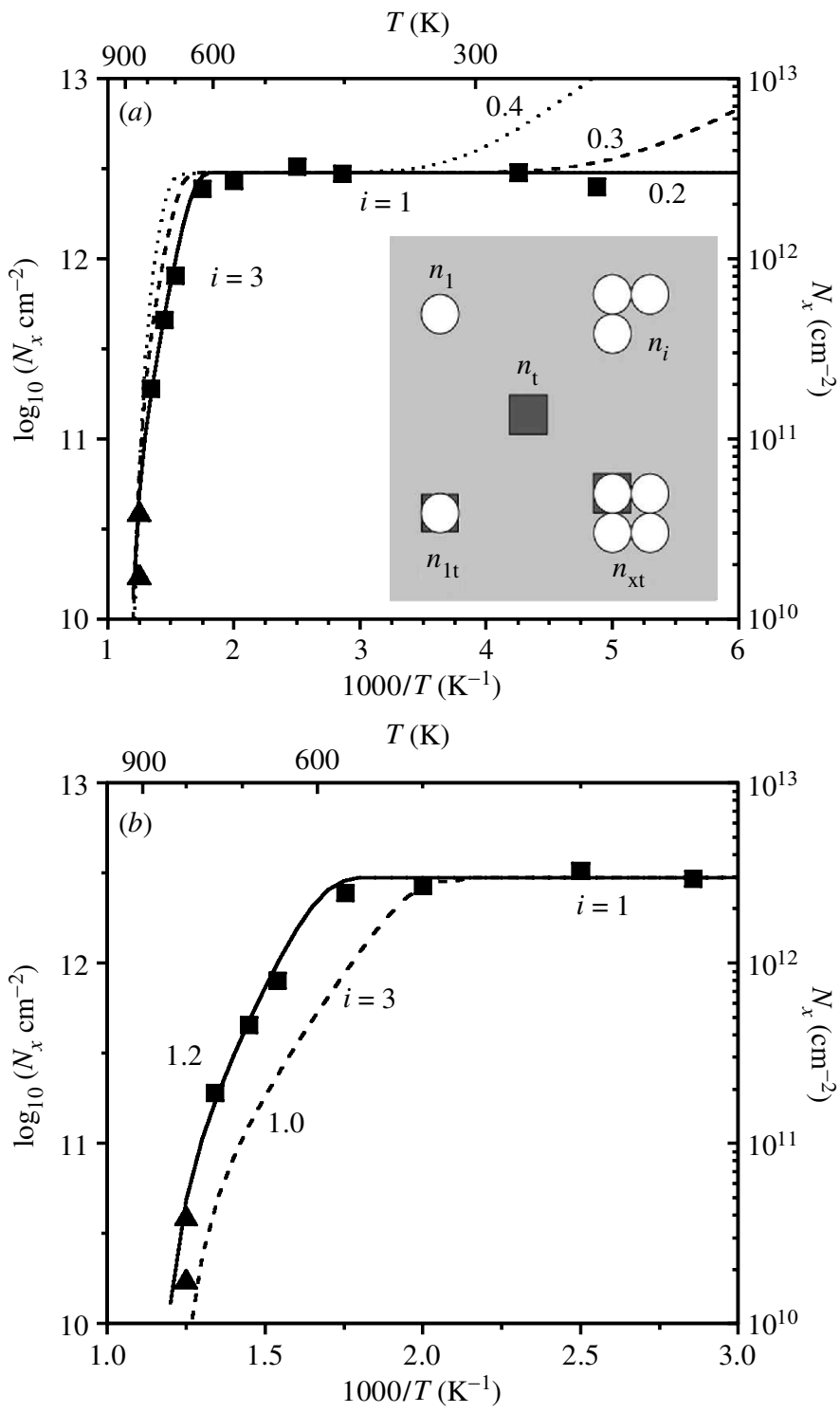

Figure 4. Arrhenius representation of Pd island density $N_{x}\left(\mathrm{~cm}^{-2}\right)$ at $0.1 \mathrm{ML}$ coverage on Ar-cleaved $\mathrm{Mg}(001)$. (a) Solid line, model for $E_{\mathrm{d}}=0.2 \mathrm{eV}, E_{\mathrm{t}}=1.5 \mathrm{eV}, E_{\mathrm{b}}=1.2 \mathrm{eV}$ and $E_{\mathrm{a}}=1.2 \mathrm{eV}$, plus curves for $E_{\mathrm{d}}=0.3 \mathrm{eV}$ (dashed line) and $0.4 \mathrm{eV}$ (dotted line), and experimental data (squares) from Haas et al. (2000). The insert shows the model for $i=3$ applicable at high temperatures, using the same notation as figure $3 a$. (b) Sensitivity to the parameter $E_{\mathrm{b}}=1.0 \mathrm{eV}$ (dashed line) and $E_{\mathrm{b}}=1.2$ (full line), with $E_{\mathrm{a}}=1.2 \mathrm{eV}$ important at high temperature, where the experimental data (triangles) indicate condensation to be incomplete. (After Venables \& Harding (2000).) See text for discussion.

density, $n_{\mathrm{t}}$, are considered here. In the RE formulation, we also need to double-up the number of coupled equations, for the densities on traps, and on the terraces between the traps. This is illustrated schematically in figure $3 a$. 
Again we can make progress by focusing on the fate of the adatoms, in this case the trapped adatoms, with density $n_{1 \mathrm{t}}$, whose fate is governed by an RE of the form

$$
\frac{\mathrm{d} n_{1 \mathrm{t}}}{\mathrm{d} t}=\sigma_{1 \mathrm{t}} D_{1} n_{1} n_{\mathrm{te}}-n_{1 \mathrm{t}} \nu_{\mathrm{d}} \exp \left(-\frac{\left(E_{\mathrm{t}}+E_{\mathrm{d}}\right)}{k T}\right),
$$

where the number of empty traps $n_{\mathrm{te}}=n_{\mathrm{t}}-n_{1 \mathrm{t}}-n_{x \mathrm{t}}$. In steady state, this equation is zero, and inserting the usual form for $D_{1} \sim \nu_{\mathrm{d}} \exp \left(-E_{\mathrm{d}} / k T\right)$, we deduce that

$$
\frac{n_{1 \mathrm{t}}}{n_{\mathrm{t}}-n_{x \mathrm{t}}}=\frac{A}{1+A}, \quad A=n_{1} C_{\mathrm{t}} \exp \left(\frac{E_{\mathrm{t}}}{k T}\right),
$$

where $C_{\mathrm{t}}$ is an entropic constant, which has been set equal to unity in the illustrative calculations performed to date. Equation (3.2) shows that the traps are full $\left(n_{1 \mathrm{t}}=n_{\mathrm{t}}-n_{x \mathrm{t}}\right)$ in the strong trapping limit, whereas they depend exponentially on $E_{\mathrm{t}} / k T$ in the weak-trapping limit, as expected. This equation is a Langmuirtype isotherm for the occupation of traps; the trapping time $\tau_{\mathrm{t}}$, in analogy to equation (2.1), which is required to reach this steady state is very short, unless $E_{\mathrm{t}}$ is very large; but if $E_{\mathrm{t}}$ is large, then all the traps are full anyway.

The total nucleation rate is the sum of the nucleation rate on the terraces and at the defects. The nucleation-rate equation without coalescence, analogous to equation $(2.2)$, is

$$
\frac{\mathrm{d} n_{x}}{\mathrm{~d} t}=\sigma_{i} D_{1} n_{1} n_{i}+\sigma_{i \mathrm{t}} D_{1} n_{1} n_{i \mathrm{t}},
$$

where the second term is the nucleation rate on defects and $n_{i \mathrm{t}}$ is the density of critical clusters attached to defects, $\sigma_{i t}$ being the corresponding capture number. In the simplest case, where the traps only act on the first atom which joins them, and entropic effects are ignored, we have

$$
A_{\mathrm{t}}=\frac{n_{1 \mathrm{t}}}{n_{1}}=\frac{\left(n_{\mathrm{t}}-n_{x \mathrm{t}}\right) A}{n_{1}(1+A)} .
$$

Typically, there are three regions: a high- $T$ region, where adatoms visit the traps but can become detached from them; a low- $T$ region, where the traps are full, but the nucleation density is largely unaffected, since $n_{x}>n_{\mathrm{t}}$; and, in between, there is a plateau region, where $n_{x}=n_{\mathrm{t}}$. The plateau is longer if $E_{\mathrm{t}}$ is higher and $E_{\mathrm{d}}$ lower. The first requirement is obvious, and the latter is required so that adatoms reach the traps before finding each other. The plateau region is interesting for fabrication, since the resulting nanostructures are independent of processing conditions. A model calculation, originally intended for $\mathrm{Fe} / \mathrm{CaF}_{2}$ (Heim et al. 1996) is shown in figure $3 b$.

This defect nucleation model contains several subcases, depending on values of the parameters. An interesting example is $\mathrm{Pd} / \mathrm{MgO}(001)$, studied with atomic-force microscopy by Haas et al. (2000), where a single set of experiments has been analysed to put bounds on four energies; these data require a high trapping energy $E_{\mathrm{t}}$ and a low value of $E_{\mathrm{d}}$, while also being sensitive to $E_{\mathrm{b}}$ and $E_{\mathrm{a}}$, as illustrated in figure 4 . In this case, the high-temperature portion of the data corresponds to the transition to $i=3$, so that individual adatoms remain attached to traps, but subsequent adatoms can become detached. These features are in agreement with calculations by Ferrari \& Pacchioni (1996) and Venables \& Harding (2000) for trapping of Pd in oxygenion vacancies. The role of surface charges in stabilizing both surface vacancies on 
insulator surfaces and small clusters attached to such point defects is very marked. Currently, different calculations agree that such effects are strong, but disagree on their exact magnitude; more comparative work is needed in this area.

Nucleation on defects has also been investigated using KMC simulations. An example is the work of Lee \& Barabási (1998), who showed that an ordered array of defect trapping centres can lead to a markedly narrower size distribution than expected for randomly nucleated islands, but only when the mean diffusion length is comparable with, not much greater than, the distance between the defect traps. This corresponds to the upper end of the plateau regime, shown here in figure $3 b$, where the adatom catchment area is roughly the same as the regular Voronoi polyhedron around each defect site. The goal of a uniform size distribution is clearly desirable for applications; it may also be aided by stress and diffusion fields as discussed in $\S \S 4$ and 5 .

\section{One- and two-dimensional rate-diffusion problems}

The previous models have assumed spatial uniformity, and diffusion effects have been included implicitly via the effect on capture numbers. There are several problems where spatial correlations (adatom-adatom or adatom-cluster) have to be considered explicitly. For that we need to solve one or more diffusion equations in the relevant one-dimensional (1D) or two-dimensional (2D) geometry, in parallel with REs.

The most obvious problem concerns the value of the capture numbers themselves. Originally it was assumed that expressions for $\sigma_{1}, \sigma_{i}$ and $\sigma_{x}$ (or $\sigma_{k}$ in general) could be written down by inspection, as simply the number of sites around the periphery of the cluster, $2 \pi\left(r_{k}+1\right)$ (Zinsmeister 1966), where the radius of the cluster in units of the jump distance is $r_{k}$. But a large effort by several authors showed that diffusion solutions, obtained by considering radial diffusion towards a typical $k$-cluster, were more realistic and had Bessel function forms with an approximately logarithmic dependence on $r_{k}$ (Venables 1973). As such, it was often assumed subsequently that capture numbers can be approximated by constant values, especially for determining the dependence of densities such as $n_{x}$ and $n_{1}$ on material parameters. But modern program suites such as MATLAB ${ }^{\mathrm{TM}}$ or MATHEMATICA ${ }^{\mathrm{TM}}$ contain library routines for Bessel functions, so it is easy to write in the correct diffusion forms. A more recent evaluation (Brune et al. 1999) has shown the extent of the differences between these forms, especially for the case of complete condensation and $i=1$, which is appropriate for STM experiments conducted at low temperatures.

Two sets of STM experiments have been conducted on the deposition and annealing of $\mathrm{Cu}$ adatoms at low temperatures (Repp et al. 2000; Knorr et al. 2002). After deposition and subsequent diffusion, the spatial distribution is not random, and this feature has been analysed quantitatively to determine the oscillatory interaction between $\mathrm{Cu}$ adatoms at a function of radial separation. In the second of these experiments, $\mathrm{Cu}$ was deposited onto a $\mathrm{Cu}(111)$ substrate at $16.5 \mathrm{~K}$, to submonolayer doses $\left(c a .3 \times 10^{-3} \mathrm{ML}\right)$, followed by annealing at various temperatures $c a .20 \mathrm{~K}$ for times up to $20 \mathrm{~min}$. At short distances, there is repulsion between adatoms, and this repulsion forms a barrier to ad-dimer formation; but once formed, dimers are completely stable and do not diffuse. This system is therefore a fascinating case: the ultimate QD, a stable cluster of two atoms on a relatively smooth surface, formed by self-assembly. No QD will ever be smaller than this! 


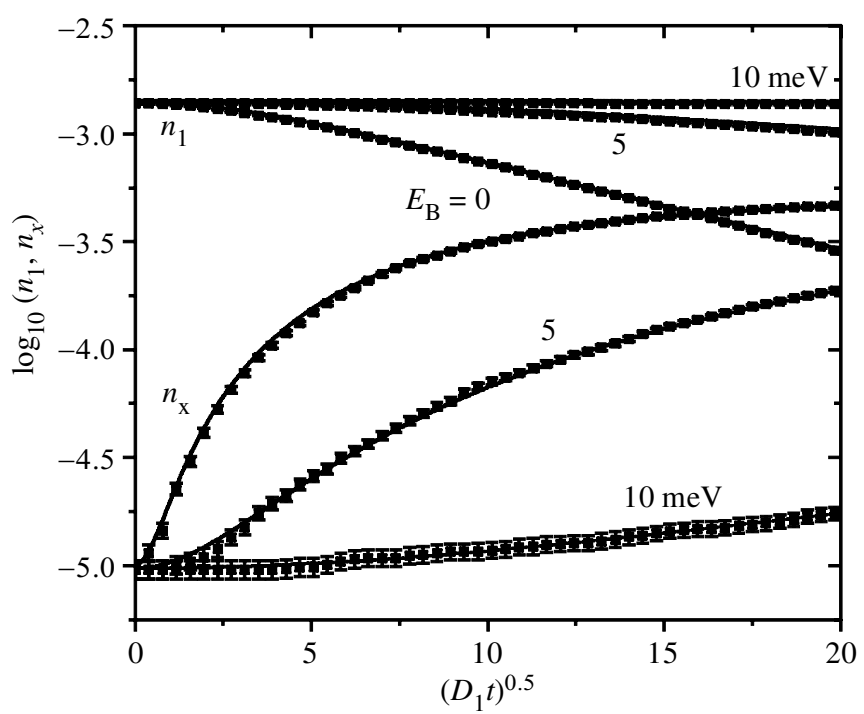

Figure 5. Predicted $n_{1}$ and $n_{x}$ annealing curves as a function of $\left(D_{1} t\right)^{0.5}$, for annealing at $16.5 \mathrm{~K}$ with attachment barriers $E_{\mathrm{B}}=0,5$ and $10 \mathrm{meV}$, compared with KMC simulations: squares with error bars, KMC; solid lines, RE integration. The capture numbers used are based on an interpolation scheme between attachment barrier and diffusion solutions, showing essential agreement with the KMC simulations. See text for discussion of how these curves apply to STM experiments on $\mathrm{Cu} / \mathrm{Cu}(111)$. (Abstracted from Venables \& Brune (2002).)

However, these experiments also test capture number models, as a repulsive barrier of height $E_{\mathrm{B}}$ changes the form of the diffusion field around adatoms and clusters, and reduces the capture number markedly if $E_{\mathrm{B}} / k T>0.2$. As shown recently (Venables $\&$ Brune 2002), the full time-dependent form of the capture numbers is required to obtain agreement between RE solutions and KMC simulations in the earliest stages of low coverage (sub-ML) annealing. The diffusion solution is almost sufficient when the barrier is zero, but for finite barriers the diffusion solution is quite wrong, and the attachment-limited (barrier) solution, $\sigma_{k}=2 \pi\left(r_{k}+1\right) \exp \left(-E_{\mathrm{B}} / k T\right)$ is much closer. Surprisingly, this is true even for barriers much smaller than the diffusion energy. Note also that this capture solution is similar to the form used by Zinsmeister (1966), but is now reduced exponentially by the Boltzmann factor for the barrier. It corresponds to the case where there are no adatom-adatom or adatom-cluster spatial correlations prior to attachment.

The full solution for annealing, appropriate to $\mathrm{Cu} / \mathrm{Cu}(111)$, is shown in figure 5 . As a result of the agreement between the KMC and RE solutions, we can extrapolate to other conditions with confidence, and we can compare these with the experimental results of Repp et al. (2000) and Knorr et al. (2002). These results showed no dimer formation during $20 \mathrm{~min}$ at $c a .17 \mathrm{~K}$ and the completion of dimer formation after 20 min at $22 \mathrm{~K}$. As a result, we were able to deduce that the barrier height $E_{\mathrm{B}}$, or alternatively the repulsive energy maximum $V_{0}$, for $\mathrm{Cu} / \mathrm{Cu}(111)$, lies between 10 and $14 \mathrm{meV}$, as illustrated in figure 6. This figure is based on an integration of the REs for each $V_{0}$, up to the end of annealing (2 or $20 \mathrm{~min}$ ) using the known $E_{\mathrm{d}}$ value, which is $40 \pm 1 \mathrm{meV}$ for $\mathrm{Cu} / \mathrm{Cu}(111)$ (Knorr et al. 2002). The comparison with KMC simulations is again excellent, but the RE computation is much faster, less than 


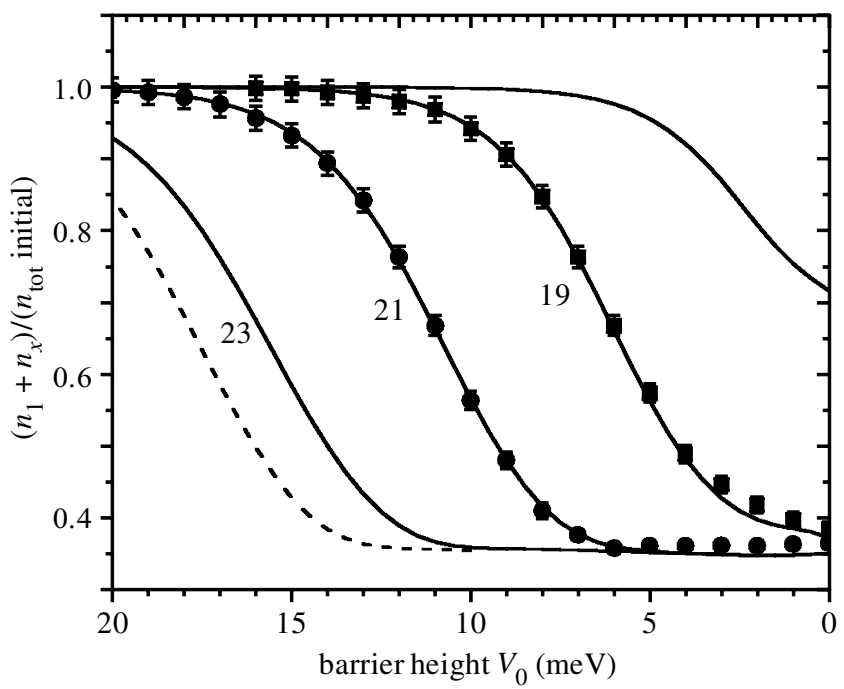

Figure 6. Predicted annealing curves as a function of barrier height $V_{0}$, at temperatures $17<T_{\mathrm{a}}<23 \mathrm{~K}$. The ratio $\left(n_{1}+n_{x}\right)$ after a $2 \mathrm{~min}$ anneal to the initial value $n_{\text {tot }}=\left(n_{1}+n_{x}\right)$ after deposition, is plotted (full lines). These curves use the time-dependent capture-number expression as in figure 5. The curves for 19 and $21 \mathrm{~K}$ are also compared with the KMC simulations (squares and circles with error bars). Additionally, a curve for annealing at $22 \mathrm{~K}$ for $20 \mathrm{~min}$ is given (dashed line). See text for discussion of how these curves apply to STM experiments on $\mathrm{Cu} / \mathrm{Cu}(111)$. (Abstracted from Venables \& Brune (2002).)

10 min for each curve, as opposed to many weeks for the KMC data. This again points to a role for RE solutions in summarizing large amounts of computation done by other methods.

There are many other problems where 1D and 2D diffusion solutions are needed to complement RE treatments. These include capture by steps, the growth of quantum wires, deposition past a mask and growth on anisotropic reconstructed surfaces. We do not discuss the first two topics; the last two are discussed next in relation to growth on (001) and (111) reconstructed-semiconductor surfaces. We consider two examples: QDs formed from Ge/Si(001), and mask deposition of $\mathrm{Ag} / \mathrm{Si}$ and $\mathrm{Ag} / \mathrm{Ge}(111)$ as prototypical metal-semiconductor deposition and annealing systems.

\section{Are semiconductors special?}

Are semiconductors special? There are many possible reasons why they might be, although in some cases this may turn on differences of degree, not of kind. Due to intense interest in device applications, each individual system has been intensively studied. Different systems that appear to be similar are often treated by specialists as being quite different. This can be traced to the interest in electronic and optical properties, with crystal-growth mechanisms only appearing as a potential obstacle to growing films with the desired properties. The most obvious distinctions made are between (i) indirect or direct band-gap materials (group IV versus III-V and II-VI); (ii) the positions of the conduction band minimum (e.g. Ge versus Si); and (iii) the magnitudes of the conduction and valence band offsets in heterostructures. 
The role of strain, and alloying or clustering, on level shifts and splitting is also very important in relation to optical properties.

From a crystal-growth viewpoint, the first complexity is the surface reconstruction. The $(2 \times 1)$ and related superstructures on $\mathrm{Si}$ and $\mathrm{Ge}(001)$ arise from the strong dimer bonds which form to reduce the number of dangling bonds. As a result, most of the sublimation energy per atom, $L$, is gained on condensation by the formation of dimers (i.e. $2 E_{\mathrm{a}}+E_{\mathrm{b} 2}$ per dimer in the notation of this paper), and very little extra energy remains to be gained when these dimers are incorporated into the growing crystal. This is consistent with the observation of a substantial density of ad-dimers at elevated temperature, with a low formation energy, $E_{\mathrm{f} 2}$, measured in quenching experiments as $E_{\mathrm{f} 2}=\left(2 L-2 E_{\mathrm{a}}-E_{\mathrm{b} 2}\right)=0.35 \pm 0.05 \mathrm{eV}$ by Tromp \& Mankos (1998).

The second complexity involves diffusion, where there are several competing mechanisms. Condensing adatoms compete to form dimers and/or join clusters, and diffusion itself is strongly anisotropic on the $(2 \times 1)$ surface, as is attachment to the different types of steps on this surface. Some of these mechanisms have been investigated quantitatively, most notably by atom-tracking STM (Swartzentruber 1996; see, for example, Venables 2000, ch. 7). Adatom diffusion energies, $E_{\mathrm{d}}$, parallel to the rows have been measured both for $\mathrm{Si}(0.67 \pm 0.08 \mathrm{eV})$ and $\mathrm{Ge}(0.62 \mathrm{eV})$, and they have also been estimated perpendicular to the rows, at $c a .1 .0 \mathrm{eV}$ and $0.95 \pm 0.1 \mathrm{eV}$, respectively. Dimer diffusion energies, $E_{\mathrm{d} 2}$, have been measured on $\mathrm{Si}(001)$, and are $c a .1 .1 \mathrm{eV}$ parallel to the rows, with higher values reported (calculated or measured) both perpendicular to the rows and in the troughs between them (Borovsky et al. 1997a, b, 1999). Thus, these surfaces present a highly complex energy landscape for diffusion, with different mechanisms active at various temperatures.

However, it is clear with the above energies that critical nuclei at practical growth temperatures may well be large, since the lateral binding (between strongly bound dimers) is so small. This feature has encouraged a 'classical' treatment of nucleation and growth in terms of edge energies for 2D nuclei; critical nucleus sizes up to $i=650$ have been deduced in some circumstances (Thies \& Tromp 1996). This approach has been reviewed recently by Tromp \& Hannon (2002). These same energies show that, although the $\mathrm{Si}$ - and $\mathrm{Ge}(001)$-growth systems may be close to $2 \mathrm{D}$ equilibrium, they are very far from equilibrium with their $(3 \mathrm{D})$ vapour, and re-evaporation is known to be negligible at normal growth temperatures, $450-650{ }^{\circ} \mathrm{C}$. Using the known sublimation energy of $\mathrm{Si}, L=4.63 \pm 0.04 \mathrm{eV}$, we can deduce from the Tromp group results that $\left(2 E_{\mathrm{a}}+E_{\mathrm{b} 2}\right)=8.91 \pm 0.07 \mathrm{eV}$. The dimer binding energy, $E_{\mathrm{b} 2}$, has been estimated theoretically as $E_{\mathrm{b} 2}=2.0 \mathrm{eV}$ (Ramstad et al. 1995), so that the adsorption energy of a $\mathrm{Si}$ adatom, $E_{\mathrm{a}}$, must have a value close to $3.5 \mathrm{eV}$.

Most interest in $\mathrm{Si}, \mathrm{Ge}$ and $\mathrm{Si}_{x} \mathrm{Ge}_{1-x}$ alloys has focused on the growth of clusters above a wetting layer, which is of the order of $3 \mathrm{ML}$ thick for pure Ge grown on $\mathrm{Si}(001)$ (Krishnamurthy et al. 1991). The growth of such clusters has been intensively studied. Clusters take the form of rectangular huts, followed by domes of various shapes, all of which are coherent with the wetting layer; when the clusters are large enough, misfit dislocations are introduced and the clusters become incoherent. For application as QDs, such clusters need to be rather small, well within the coherent size limit. It has been found that the deposition temperature and flux play key roles, not only in determining nucleation densities but also in determining the extent of 

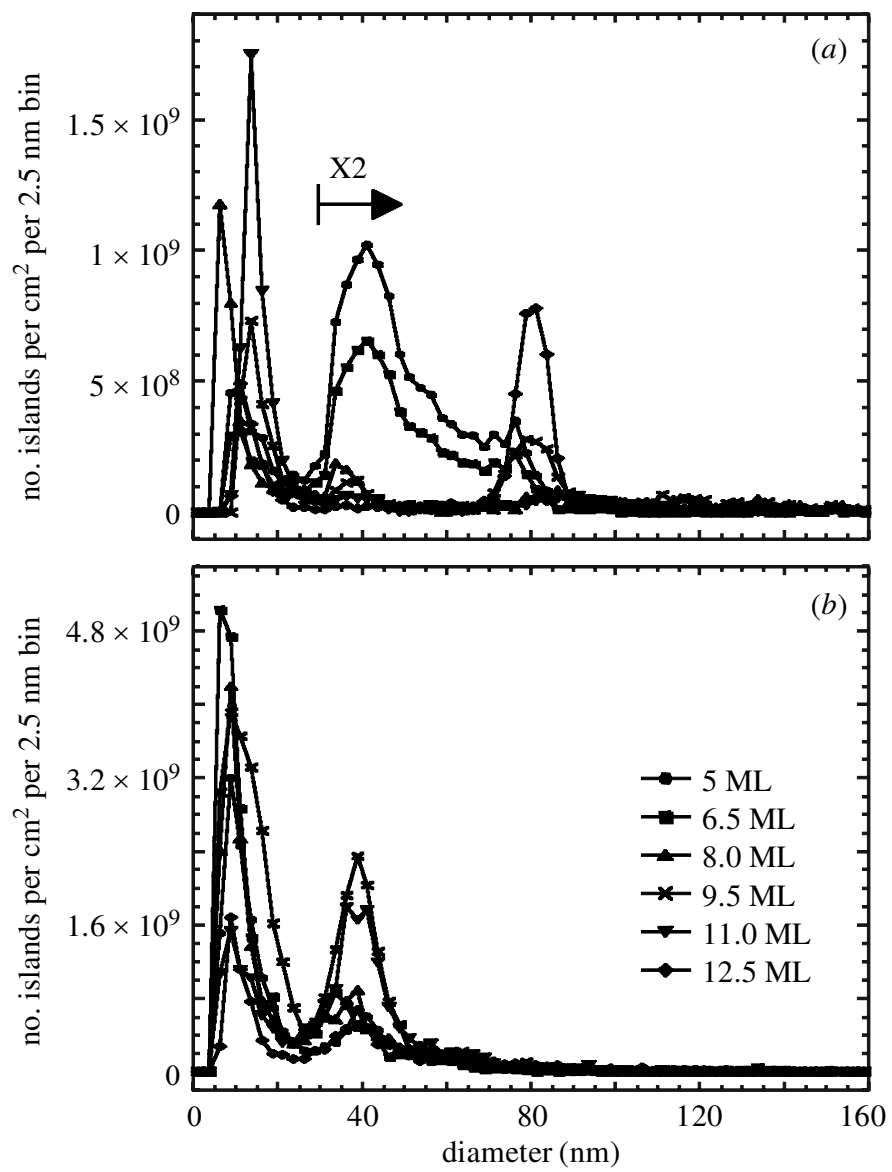

Figure 7. Size distributions of $\mathrm{Ge} / \mathrm{Si}(001)$ islands grown at $(a) 600{ }^{\circ} \mathrm{C}$ and $(b) 450{ }^{\circ} \mathrm{C}$ to the coverages indicated. For each temperature, the left-hand peak corresponds to huts, while the right-hand peak corresponds to domes. At $600{ }^{\circ} \mathrm{C}$ and $650{ }^{\circ} \mathrm{C}$ (not shown), large alloyed hut peaks exist between the two outer peaks; these large hut peaks are not present at $550{ }^{\circ} \mathrm{C}$ (not shown) and $450^{\circ} \mathrm{C}$. Note also that the dome peak shifts to larger sizes at higher growth temperatures. The existence of large, alloyed huts and the shift of the dome peak to larger sizes is indicative of Si interdiffusion. Formation of a lower misfit alloy allows clusters to attain larger sizes prior to shape transitions of dislocation introduction. (After Chaparro et al. (2000a).)

surface diffusion and interdiffusion leading to alloying in the surface and subsurface regions.

Recently, very specific effects of the role of stress on diffusion and interdiffusion have been demonstrated in these systems. First, island formation is a response to the $4.2 \%$ mismatch between the Ge and $\mathrm{Si}$ lattice constants (less in the $\mathrm{Si}_{x} \mathrm{Ge}_{1-x}$ alloy system). Second, there is a large compressive stress, or equivalently elastic energy, at the edge of Ge-rich islands on the wetting layer. This region therefore has a higher chemical potential for diffusing Ge adatoms or dimers, which is higher for larger islands. At a high enough growth temperature, this extra potential results in trench formation around domes, in which not only Ge but also Si diffuses away from the high-stress region (Chaparro et al. 2000a). 


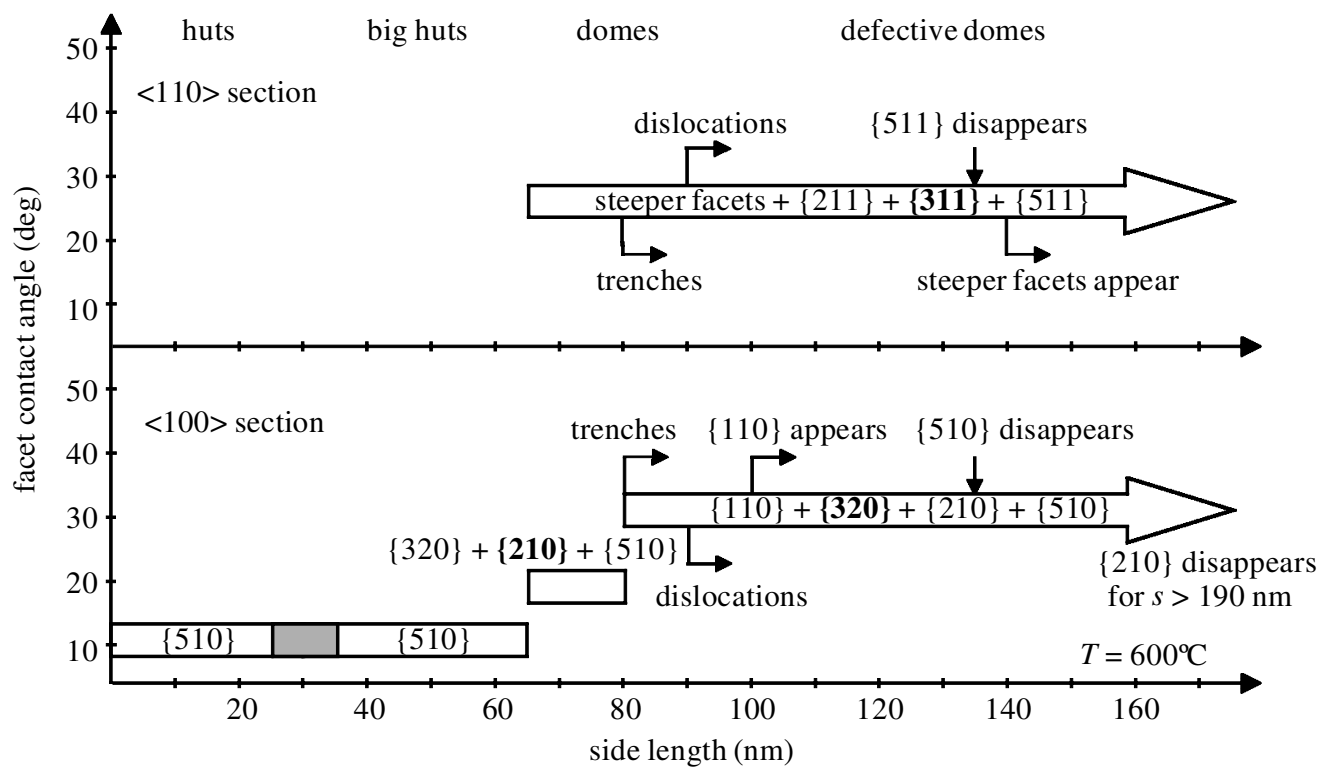

Figure 8. Summary of shape evolution of Ge clusters grown at $F=1.4 \mathrm{ML} \mathrm{min}^{-1}$ onto $\mathrm{Si}(001)$ at $600{ }^{\circ} \mathrm{C}$. The vertical position of the horizontal bars represents the contact angle of the dominant facet with the (001) substrate. The horizontal extent of the bar represents the size range over which that morphology exists. The top half of the figure shows the evolution of the $\langle 110\rangle$ cross-section and the bottom half the $\langle 100\rangle$ cross-section. (After Chaparro et al. (2000a).)

Stress relief via interdiffusion also accounts for certain features of the hut-dome transition and the dome size distributions shown in figure 7 . At $450{ }^{\circ} \mathrm{C}$ the peak at $40 \mathrm{~nm}$ diameter corresponds to coherent domes, and the peak at smaller sizes to huts, but interdiffusion is not an important factor. At $600{ }^{\circ} \mathrm{C}$, the dome peak has shifted out to $80 \mathrm{~nm}$ diameter, while a new broad peak has appeared centred at $40 \mathrm{~nm}$, which corresponds to partly alloyed huts. This hut-dome transition can thus be delayed to some extent via alloying at the higher growth temperatures. The sequence of shapes, the formation of trenches, and the introduction of dislocations have been documented in detail (Chaparro et al. 2000a) at a series of growth temperatures; the sequence at $600{ }^{\circ} \mathrm{C}$ is shown in figure 8 .

We are presently quite a long way from a fully quantitative model of all competing effects in this and similar systems: nucleation, growth (initial and stress limited), ripening or coarsening, shape fluctuations and transitions, stress-influenced interdiffusion, and so on. But many pieces of the argument are in place. The high ad-dimer concentration and the small dimer-dimer interaction relates to the high critical nucleus size (small supersaturation) for nucleation and initial growth (Tromp \& Hannon 2002). The high density of mobile species makes both Ostwald ripening and shape fluctuations relatively easy, as seen directly by in situ low-energy electron microscopy (Ross et al. 1998). Interdiffusion is strongly influenced by high stress concentrations at the edge of the clusters, and this effect leads to the trenches, with an effective diffusion coefficient that is considerably faster than bulk diffusion (Chaparro et al. 2000b; Denker et al. 2001).

Similar considerations apply to growth on $\mathrm{Si}(111)$, but here the reconstruction is the famous $(7 \times 7)$ structure, whose symmetry is triangular with a repeat distance of 
$2.688 \mathrm{~nm}$. Adatoms form the mobile species, and the surface is rougher than (001), in the sense that there is a deep hole at the corners of the $(7 \times 7)$ cell and troughs along the dimerised edges, which separate the faulted and unfaulted halves of the cell. Thus, diffusion on this surface represents a serious obstacle course; moreover, growth of a second material, whether of a semiconductor such as Ge or metal such as $\mathrm{Ag}$, reconstructs the surface differently. In the latter case the $\sqrt{3}$ (i.e. $\sqrt{3} \times \sqrt{3} R 30^{\circ}$ ) structure with ca. $1 \mathrm{ML}$ coverage is especially stable, and growth and annealing at temperatures above $200{ }^{\circ} \mathrm{C}$ take place on that surface, which is in effect an interface compound. Direct integration of the REs for Ag adatoms on both Si and Ge(111) has been used to obtain accurate values of adsorption, diffusion and binding energies on these surfaces (Venables et al. 1997), but again there are details left to sort out, notably those concerned with small particle mobility and interdiffusion.

At lower temperatures there are kinetic limitations to the formation of the $\sqrt{3}$ structure, and this offers the possibility of manipulating the kinetics to obtain various types of QDs on the scale of the $(7 \times 7)$ structure. For example, several metal deposits on $\mathrm{Si}(111)$ have been investigated by Wang \& Lai (2001) and other authors; silicide nucleation and growth have been shown to be different on the faulted and unfaulted halves of the $7 \times 7$ unit cell (Bennett et al. 1994; Bennett \& von Känel 1999). It remains to be seen whether such structures can be developed further or analysed effectively using the methods described here.

\section{Discussion and conclusions}

A brief survey has been given of the use of rate and rate-diffusion equations to analyse experiments on nucleation densities during deposition and annealing. Extension of the simplest model to include nucleation on point defects is described, and computing accurate capture numbers has been revisited in comparison with KMC simulations. Experimental examples include metal-insulator, metal-metal, metal-semiconductor and elemental semiconductor-growth systems, including those described as QDs. The growth of III-V and II-VI compound semiconductors has not been described, but these involve all the complexities discussed here, plus others of their own. In particular, the stages of surface reaction, partial interdiffusion leading to composition variation within the dot, are important in obtaining agreement with optical properties in III-V compounds (e.g. Shumway et al. 2002). An excellent example of multi-layer stacked QDs is in II-VI compounds (e.g. Raab et al. 2002), where impressive size and spatial uniformity has been demonstrated. These are good indicators of where the field is going, but are beyond the scope of this paper.

One interesting item for future work is a comparison of these semiconductor and metal-semiconductor systems with the metal-metal systems discussed in $\S 5$. From the point of view of a large-scale description via REs, they are quite similar. The role of the stress is to change the energy landscape near the edge of the clusters, and so to reduce the capture number for larger clusters. If the energies in the problem scale with the temperatures used in growth and annealing, and $600{ }^{\circ} \mathrm{C}(873 \mathrm{~K})$ for $\mathrm{Ge} / \mathrm{Si}(001)$ is divided by a factor of 40 , then we find we are at $21.8 \mathrm{~K}$, in the range of temperatures explored during annealing of $\mathrm{Cu} / \mathrm{Cu}(111)$ in figure 5 . The value of $E_{\mathrm{d}}$ for $\mathrm{Cu} / \mathrm{Cu}(111)$ is $40 \mathrm{meV}$, and $40 \times(40 \mathrm{meV})=1.6 \mathrm{eV}$, somewhat higher, but of the same order of magnitude as that expected for Ge surface diffusion, and probably quite similar for diffusion within the first layer or two. 
The potential barrier for adatom attachment interpreted in $\S 4$ as $12 \pm 2 \mathrm{meV}$ for $\mathrm{Cu} / \mathrm{Cu}(111)$ (Venables \& Brune 2002) translates into $0.48 \pm 0.08 \mathrm{eV}$ on multiplying by 40 to achieve the same effect in $\mathrm{Ge} / \mathrm{Si}(001)$ at the higher temperature, all other things being equal. That, of course, they are not, and factors of 40 in temperature are extreme extrapolations. Nonetheless, the simple calculations of the energy landscape at the edge of QDs that have so far been performed give energies ca. $0.2-0.4 \mathrm{eV}$ (Drucker 1993; Barabási 1997; Enomoto 2001). On the other hand, the binding energy of dimer pairs, expected to be below $0.35 \mathrm{eV}$ for $\mathrm{Ge}_{2}-\mathrm{Ge}_{2}$ on $\mathrm{Si}(001)$, becomes less than $10 \mathrm{meV}$ on dividing by 40, in contrast to a binding energy of the order of $0.3 \mathrm{eV}$ expected for $\mathrm{Cu}$ adatom pairs. For example, Ovesson et al. (2001) have been interested in similar phenomena to those discussed in $\S 4$, and they calculated that $E_{\mathrm{b}}=0.26 \mathrm{eV}$ for $\mathrm{Cu}$ pairs on $\mathrm{Cu}(111)$. There are clearly other possible bases for comparison between such systems, which can be explored in future work.

There are also analogies between silicide nucleation on $\mathrm{Si}$ and $\mathrm{Ge}(111)$ and metalmetal growth, e.g. $\mathrm{Ag} / \mathrm{Ag}(111)$ and $\mathrm{Ag} / \mathrm{Ag}(2 \mathrm{ML}) / \mathrm{Pt}(111)$, where interface layers show misfit dislocation arrays, which act as a barrier to diffusion, and faulted or unfaulted halves of the surface cell, which act as preferential nucleation sites (Brune et al. 1998). The main difference between these widely different systems seems to be the large difference in critical nucleus size or, equivalently, the tendency to Ostwald ripening during deposition and annealing, due to the relative difference of the lateral pair binding energy of the diffusing species. These are all examples of quantities which can be easily varied, and explored rapidly with an RE treatment, and they represent cases which may merit further study.

\section{References}

Barabási, A.-L. 1997 Self-assembled island formation in heteroepitaxial growth. Appl. Phys. Lett. 70, 2565-2567.

Bennett, P. A. \& von Känel, H. 1999 Scanning tunneling microscopy of silicides. J. Phys. D 32, R71-R87.

Bennett, P. A., Cahill, D. G. \& Copel, M. 1994 Interstitial precursor to silicide formation on Si(111)- $(7 \times 7)$. Phys. Rev. Lett. 73, 452-455.

Borovsky, B., Kruger, M. \& Ganz, E. 1997a Diffusion of the silicon dimer on Si(001): new possibilities at $450 \mathrm{~K}$. Phys. Rev. Lett. 78, 4229-4232.

Borovsky, B., Kruger, M. \& Ganz, E. $1997 b$ Diffusion of adsorbed Si dimers on Si(001). Surf. Sci. 385, 146-154.

Borovsky, B., Kruger, M. \& Ganz, E. 1999 Piecewise diffusion of the silicon dimer. Phys. Rev. B 59, 1598-1601.

Brune, H. 1998 Microscopic view of epitaxial metal growth: nucleation and aggregation. Surf. Sci. Rep. 31, 121-229.

Brune, H., Giovannini, M., Bromann, K. \& Kern, K. 1998 Self-organized growth of nanostructure arrays on strain-relief patterns. Nature 394, 451-453.

Brune, H., Bales, G. S., Jacobsen, J., Boragno, C. \& Kern, K. 1999 Measuring surface diffusion from nucleation island densities. Phys. Rev. B 60, 5991-6006.

Chaparro, S. A., Zhang, Y., Drucker, J., Chandrasekhar, D. \& Smith, D. J. 2000 a Evolution of $\mathrm{Ge} / \mathrm{Si}(100)$ islands: island size and temperature dependence. J. Appl. Phys. 87, 2245-2254.

Chaparro, S. A., Zhang, Y. \& Drucker, J. $2000 b$ Strain relief via trench formation in Ge/Si(100) islands. Appl. Phys. Lett. 76, 3534-3536. 
Denker, U., Schmidt, O. G., Jin-Philipp, N.-Y. \& Eberl, K. 2001 Trench formation around and between self-assembled Ge islands on Si. Appl. Phys. Lett. 78, 3723-3725.

Drucker, J. 1993 Coherent islands and microstructural evolution. Phys. Rev. B 48, 18 203-18 206.

Ehrlich, G. 1991 Direct observation of the surface diffusion of atoms and clusters. Surf. Sci. 246, 1-12.

Ehrlich, G. 1994 Diffusion of individual adatoms. Surf. Sci. 299-300, 628-642.

Enomoto, Y. 2001 Coarsening dynamics of self-assembled quantum dots with elastic field interactions. Surf. Sci. 493, 585-590.

Ferrari, A. M. \& Pacchioni, G. 1996 Metal deposition on oxide surfaces; a quantum-chemical study of the interaction of $\mathrm{Rb}, \mathrm{Pd}$ and $\mathrm{Ag}$ atoms with the surface vacancies of MgO. J. Phys. Chem. 100, 9032-9037.

Haas, G., Menck, A. Brune, H., Barth, J. V., Venables, J. A. \& Kern, K. 2000 Nucleation and growth of supported clusters at defect sites: Pd/MgO (001). Phys. Rev. B 61, 11 105-11 108.

Heim, K. R., Coyle, S. T., Hembree, G. G., Venables, J. A. \& Scheinfein, M. 1996 Growth of nanometer-size metallic particles on $\mathrm{CaF}_{2}$ (111). J. Appl. Phys. 80, 1161-1170.

Kellogg, G. L. 1994 Field ion microscope studies of single-atom surface diffusion and cluster nucleation on metal surfaces. Surf. Sci. Rep. 21, 1-88.

Knorr, N., Brune, H., Epple, M., Hirstein, A., Schnieder, M. A. \& Kern, K. 2002 Long-range adsorbate interactions mediated by a two-dimensional electron gas. Phys. Rev. B 65, 115420 .

Krishnamurthy, M., Drucker, J. S. \& Venables, J. A. 1991 Microstructural evolution during the heteroepitaxy of Ge on vicinal Si(100). J. Appl. Phys. 69, 6461-6471.

Lee, C. \& Barabási, A.-L. 1998 Spatial ordering of islands grown on patterned surfaces. Appl. Phys. Lett. 73, 2651-2653.

McDaniels, T. H., Venables, J. A. \& Bennett, P. A. 2001 Island nucleation in a reactive twocomponent system. Phys. Rev. Lett. 87, 176105.

Ovesson, S., Bogicevic, A., Wahnström, G. \& Lundqvist, B. I. 2001 Neglected adsorbate interactions behind diffusion prefactor anomalies on metals. Phys. Rev. B 64, 125423.

Petersen, M., Ratsch, C., Caflisch, R. E. \& Zangwill, A. 2001 Level set approach to reversible epitaxial growth. Phys. Rev. E 64, 061602.

Raab, A., Lechner, R. T. \& Springholz, G. 2002 Self-organized lateral ordering for vertically aligned PbSe/PbEuTe quantum dot superlattices. Appl. Phys. Lett. 80, 1273-1275.

Ramstad, A., Brocks, G. \& Kelly, P. J. 1995 Theoretical study of the Si(100) surface reconstruction. Phys. Rev. B 51, 14504-14523.

Ratsch, C., Gyure, M. F., Caflisch, R. E., Gibou, F., Petersen, M., Garcia, J., Kang, M. \& Vvedensky, D. D. 2002 Level set method for island dynamics in epitaxial growth. Phys. Rev. B 65, 195403.

Repp, J., Moresco, F., Meyer, G., Rieder, K.-H., Hyldgaard, P. \& Persson, M. 2000 Substrate mediated long-range oscillatory interaction between adatoms: $\mathrm{Cu} / \mathrm{Cu}(111)$. Phys. Rev. Lett. 85, 2981-2984.

Ross, F. M., Tersoff, J. \& Tromp, R. M. 1998 Coarsening of self-assembled Ge quantum dots on Si(001). Phys. Rev. Lett. 80, 984-987.

Shumway, J., Williamson, A. J., Zunger, A., Passaseo, A., DeGorgi, M., Cingolani, R., Catalano, M. \& Crozier, P. 2002 Electronic structure consequences of In/Ga composition variations in self-assembled $\operatorname{In}_{x} \mathrm{Ga}_{1-x} \mathrm{As} / \mathrm{GaAs}$ alloy quantum dots. Phys. Rev. B 64, 125302.

Swartzentruber, B. S. 1996 Direct measurement of surface diffusion using atom-tracking scanning tunneling microscopy. Phys. Rev. Lett. 76, 459-462.

Thies, W. \& Tromp, R. M. 1996 Nucleation in Si(001) homoepitaxial growth. Phys. Rev. Lett. 76, 2770-2773.

Tromp, R. M. \& Hannon, J. B. 2002 Thermodynamics of nucleation and growth. Surf. Rev. Lett. 9, 1565-1593. 
Tromp, R. M. \& Mankos, M. 1998 Thermal adatoms on Si(001). Phys. Rev. Lett. 81, 1050-1053. Tsong, T. T. 1990 Atom-probe field ion microscopy. Cambridge University Press.

Venables, J. A. 1973 Rate equation approaches to thin film nucleation kinetics. Phil. Mag. 27, $697-738$.

Venables, J. A. 1987 Nucleation calculations in a pair-binding model. Phys. Rev. B 36, 41534162.

Venables, J. A. 1994 Atomic processes in crystal growth. Surf. Sci. 299-300, 798-817.

Venables, J. A. 2000 Introduction to surface and thin film processes, ch. 4-5. Cambridge University Press.

Venables, J. A. \& Brune, H. 2002 Capture numbers in the presence of repulsive adsorbate interactions. Phys. Rev. B 66, 195404.

Venables, J. A. \& Harding, J. H. 2000 Nucleation and growth of supported metal clusters at defect sites on oxide and halide (001) surfaces. J. Cryst. Growth 211, 27-33.

Venables, J. A., Metcalfe, F. L. \& Sugawara, A. 1997 Competing processes and controlling energies at the $\mathrm{Ag} / \mathrm{Ge}(111)$ interface. Surf. Sci. 371, 420-430.

Wang, Y. L. \& Lai, M. Y. 2001 Formation of surface magic clusters: a pathway to monodispersed nanostructures on surfaces. J. Phys. Condens. Matter 13, R589-R618.

Zinsmeister, G. 1966 A contribution to Frenkel's theory of condensation. Vacuum 16, 529-535.

\section{Discussion}

P. Dobson (Department of Engineering Science, University of Oxford, UK). Perhaps this is an unfair question, but as a representative of both the epitaxial layer and the quantum dot community: what have epitaxial quantum dots to offer to compete with the colloidal route? For example, epitaxial quantum dots are badly defined in size, shape, composition and spacing, etc., whereas the colloids are better in all these ways.

J. A. Venables. This type of question arises as soon as one starts to think about technological applications in the medium term. There may always be another innovation, developed via a completely different route, which can blow one away in the marketplace! But that innovation may have other disadvantages, and most of the time we scientists are testing out our own approaches, in a relatively far-from-market environment, and pursuing our own hunches.

In particular, an obvious advantage of the epitaxial approach is that integration with bulk silicon device and interconnect technology is standard; doubtless those who advocate the colloid approach are working hard to overcome any limitations in this regard. But we should emphasize that the uniformity achieved is impressive, particularly in stacked multilayer QDs (e.g. Raab et al. 2002), and there is no indication that the community is at the end of that road. Another technological question is 'how uniform is uniform enough?' We would expect the answer to depend on the application envisaged, but it seems that the present devices are quite good enough for light-emitting diodes and the best are good enough for lasers.

R. A. Oliver (Department of Materials, University of Oxford, UK). You discussed kinetic models that explored the growth of QDs on semiconductor surfaces. However, you also mentioned that in heteroepitaxial semiconductor growth a quasiequilibrium exists at least in two dimensions. Would the you like to comment on the thermodynamic equilibrium models of Stranski-Krastanov (S-K) growth devised by Shchukin and others? Are these models inherently in tension with the kinetic 
models, or are both useful abstractions, which could be used in tandem to further our understanding?

J. A. Venables. Crystal nucleation and growth are out-of-equilibrium phenomena, but within the models there is interplay between thermodynamic and kinetic reasoning. Often the thermodynamic (or statistical mechanical) reasoning is applied in a spatially or temporally localized sense, so that one can approximate the situation by a local equilibrium, within which the rates of forward and back reactions are almost equal. For example, the reactions on the left-hand side of figure 1 are in local equilibrium in this sense, enabling us to write $n_{i}$ in terms of $n_{1}$ and the cluster binding energy $E_{i}$ in equation $(2.2)$.

There are many current attempts, by Barabási, Drucker, Shchukin and others, to incorporate strain into the description of S-K growth, and to investigate the effect of strain on the overall kinetics. Barabási and Shchukin have perhaps gone furthest in the direction of claiming that strain produces equilibrium structures, but others hotly dispute equilibrium arguments. These other authors (Drucker 1993; Ross et al. 1998) have maintained that these are growth effects, where Ostwald ripening (coarsening) may be slowed down by strain, but that we are dealing with at most local energy minima along a long-term annealing path. Our feeling is that the latter view is correct, but until such arguments get sorted out they can generate a lot of heat as well as light, and that is where we are right now; maybe the really crucial experiment still needs to be done?

A. M. Stoneham (Department of Physics and Astronomy, University College London, $U K$ ). Could you comment on the role of long-range forces? For non-conducting substrates, electrostatic forces (and energies) are quite big, and one would expect them to affect observed dot pair-distribution functions (indeed for elastic interaction these have been seen dynamically).

J. A. Venables. There are certainly several types of interaction potentials that have long-range tails, and not only for non-conducting substrates. For example, the $\mathrm{Cu} / \mathrm{Cu}(111)$ case described in $\S 4$ of the paper is thought to show oscillatory longrange surface state interactions, which vary as $r^{-2}$ at large $r$. The Friedel oscillations give preferred-pair separations, which are seen in STM images taken at low temperatures. As you suggest, elastic interactions, and especially electrostatic interactions in, or on, insulators, are often important. For example, ionic interactions between charged point defects and charged adatoms might be expected to have potential tails varying as $r^{-1}$. However, the importance of such potential tails will vary markedly, depending on the experimental temperature. The $\mathrm{Cu} / \mathrm{Cu}(111)$ and other "smooth surface' phenomena could only be demonstrated by operating at a suitably low temperature, such that the barrier energies are a substantial fraction of $k T$. 